\title{
Survey of radiation field and dose in human papillomavirus- positive oropharyngeal cancer: is de-escalation actually applied in clinical practice?
}

\author{
Kyu Hye Choi ${ }^{1}$, Jin Ho Song ${ }^{1}$, Yeon-Sil Kim ${ }^{1}$, Sung Ho Moon ${ }^{2}$, Jeongshim Lee ${ }^{3}$, Young-Taek Oh${ }^{4}$, Dongryul Oh${ }^{5}$, \\ Jin Ho Kim ${ }^{6}$, Jun Won Kim ${ }^{7}$ \\ ${ }^{1}$ Department of Radiation Oncology, Seoul St. Mary's Hospital, College of Medicine, The Catholic University of Korea, Seoul, Korea \\ ${ }^{2}$ Proton Therapy Center, Research Institute and Hospital, National Cancer Center, Goyang, Korea \\ ${ }^{3}$ Department of Radiation Oncology, Inha University Hospital, Inha University School of Medicine, Incheon, Korea \\ ${ }^{4}$ Department of Radiation Oncology, Ajou University Hospital, Ajou University School of Medicine, Suwon, Korea \\ ${ }^{5}$ Department of Radiation Oncology, Samsung Medical Center, Sungkyunkwan University School of Medicine, Seoul, Korea \\ ${ }^{6}$ Department of Radiation Oncology, Seoul National University Hospital, Seoul National University College of Medicine, Seoul, Korea \\ ${ }^{7}$ Department of Radiation Oncology, Gangnam Severance Hospital, Yonsei University College of Medicine, Seoul, Korea
}

Received: May 24, 2021

Revised: July 1, 2021

Accepted: July 8, 2021

\section{Correspondence:}

Yeon-Sil Kim

Department of Radiation Oncology,

Seoul St. Mary's Hospital, College of

Medicine, The Catholic University of

Korea, 222 Banpo-daero, Seocho-gu,

Seoul 06591, Korea

Tel: +82-2-2258-6259

E-mail: yeonkim7@catholic.ac.kr ORCID:

https://orcid.org/0000-0003-1291-0821
Purpose: Studies on de-escalation in radiation therapy (RT) for human papillomavirus-positive $(\mathrm{HPV}(+))$ oropharyngeal cancer (OPC) are currently ongoing. This study investigated the current practice regarding the radiation dose and field in the treatment of HPV(+) OPC.

Materials and Methods: The Korean Society for Head and Neck Oncology conducted a questionnaire on the primary treatment policy. Among them, for $\mathrm{HPV}(+)$ OPC scenarios, radiation oncologists were questioned regarding the field and dose of RT.

Results: Forty-two radiation oncologists responded to the survey. In definitive concurrent chemoradiotherapy (CCRT) treatment for stage T2N1M0 OPC, most respondents prescribed a dose of $>60 \mathrm{~Gy}$ to the primary tonsil and involved ipsilateral lymph nodes. However, eight of the respondents prescribed a relatively low dose of $\leq 54$ Gy. For stage T2N1M0 OPC, postoperative adjuvant RT was prescribed by eight and nine respondents with a lower dose of $\leq 50$ Gy for the ipsilateral tonsil and involved neck, respectively. In definitive CCRT in complete remission after induction chemotherapy for initial stage T2N3M0 OPC, de-escalation of the tonsil and involved neck were performed by eight and seven respondents, respectively. Regarding whether de-escalation is applied in radiotherapy for $\mathrm{HPV}(+)$ OPC, 27 (64.3\%) did not do it at present, and 15 (35.7\%) were doing or considering it.

Conclusion: The field and dose of prescribed treatment varied between institutions in Korea. Among them, dose de-escalation of RT in HPV(+) OPC was observed in approximately $20 \%$ of the respondents. Consensus guidelines will be set in the near future after the completion of ongoing prospective trials.

Keywords: Oropharyngeal neoplasms, Human papillomavirus, Radiotherapy, De-escalation

\section{Introduction}

Head and neck cancer (HNC) is the 6th most common cancer worldwide and is known to be associated with smoking and heavy alcohol consumption [1]. Recently, human papillomavirus (HPV) infection has been identified as an independent causative factor in the development of HNC, and HPV-related "HPV(+)" HNC is known to have a different course compared with HPV-unrelated "HPV(-)"

(C) 2021 The Korean Society for Radiation Oncology

This is an Open Access article distributed under the terms of the Creative Commons Attribution Non-Commercial License (http://creativecommons.org/licenses/by-nc/4.0/) which permits unrestricted non-commercial use, distribution, and reproduction in any medium, provided the original work is properly cited. 
HNC $[2,3]$. The incidence of HPV $(+)$ oropharyngeal cancer (OPC) has been increasing since the 1990s, from $40.5 \%$ before 2000 to $72.2 \%$ after $2000[4,5]$. HPV(+) OPC exhibits a pandemic-like worldwide distribution, and a good overall survival (OS) compared with HPV(-) OPC has been reported in several previous prospective studies and meta-analyses [6-12].

$\mathrm{HPV}(+)$ OPC shows a high response rate to radiation therapy (RT) and chemotherapy and has a good prognosis, therefore, many studies on the de-escalation of treatment are ongoing [13-15]. However, the paradigm for treatment has not yet changed, and the results of these studies are also controversial. There are needs to know current clinical practice before the results of ongoing prospective trials provide clear evidence. The purpose of this study was to investigate the current practice regarding the radiation dose and field in the treatment of $\mathrm{HPV}(+) \mathrm{OPC}$.

\section{Materials and Methods}

Head and neck oncology experts at the Korean Society for Head and Neck Oncology (KSHNO) were surveyed from July to August 2019. Five scenarios for OPC treatment were developed, and the subcommittee on Oropharyngeal Cancer Treatment Guidelines of the KSHNO conducted a questionnaire on primary treatment policy [16]. Radiation oncologists were further investigated regarding the field and dose of RT prescribed in the case of definitive and adjuvant $\mathrm{RT}$, and definitive concurrent chemoradiotherapy (CCRT) in radiologic complete remission after induction chemotherapy (IC). The questionnaire was sent via e-mail twice, and the results were collected and analyzed in October 2019.

The scenario for definitive CCRT was the case of a 52-year-old female non-smoker who was diagnosed with stage T2N1M0 $\mathrm{HPV}(+)$ right tonsillar squamous cell carcinoma (SCC) without base of tongue invasion and with right II-III cervical lymph node (LN) metastasis (Fig. 1A). As the second question in the same scenario, it was assumed that surgical resection of the right tonsil with neck dissection (right modified radical neck dissection and left selective neck dissection) was performed, and adjuvant RT was planned in an intermediate-risk group with no adverse features other than two metastatic LNs.

The third scenario was of a 69-year-old non-smoker who was diagnosed with stage T2N3M0 HPV(+) left tonsillar SCC with metastasis to the ipsilateral upper, middle, and lower internal jugular chain (Fig. 1B). This patient received two cycles of IC comprising docetaxel, cisplatin, and 5-fluorouracil, and follow-up imaging showed clinically complete remission after neoadjuvant treatment (ycCR) (Fig. 1C). The radiation field and dose in definitive CCRT in this case were investigated.

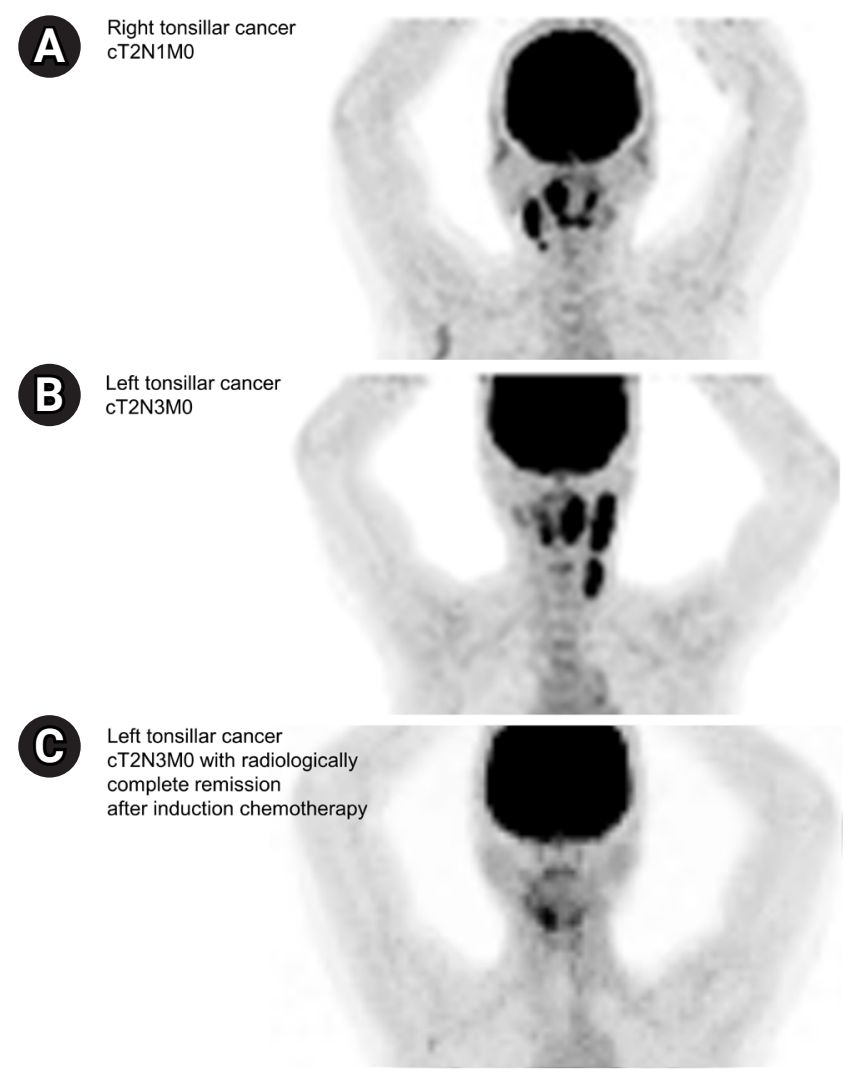

Fig. 1. Imaging studies of (A) the T2N1M0 right tonsillar cancer case and $(B)$ initial T2N3M0 left tonsillar cancer case and (C) radiologic response after induction chemotherapy.

Each respondent was asked whether de-escalation of the field or dose of RT was performed in the current clinical treatment of $\mathrm{HPV}(+)$ OPC. In addition, a detailed analysis was conducted to investigate how to reduce the radiation dose and field in each scenario. Statistical analyses were performed using SPSS version 24 (IBM, Armonk, NY, USA). This study was a survey study, not a study on human subjects; therefore, it is not applicable in terms of ethical considerations.

\section{Results}

\section{Surveyed population}

Radiation oncologists from 42 institutions responded to the questionnaire. The response rate was 56\% (42/75 institutions). The distribution of respondents was $13(86.7 \%)$ of 15 institutions with $>1,000$ beds, 28 (48.3\%) of 58 with 500-1,000 beds, and one $(50 \%)$ of two institutions with $<500$ beds. The characteristics of the 42 respondents are summarized in Table 1.

\section{Definitive CCRT in T2N1M0}

The response regarding the radiation field for definitive CCRT in right 
Table 1. Characteristics of respondents $(n=42)$

\begin{tabular}{lc}
\hline Characteristic & Number (\%) \\
\hline Board certification (yr) & $5(11.9)$ \\
$<5$ & $11(26.2)$ \\
$\geq 5$ and $<10$ & $11(26.2)$ \\
$\geq 10$ and $<20$ & $15(35.7)$ \\
$\geq 20$ & \\
Average annual patients & $35(83.3)$ \\
$<50$ & $6(14.3)$ \\
$\geq 50$ and $<100$ & $1(2.4)$ \\
$\geq 100$ & \\
Hospital beds & $15(35.7)$ \\
$\geq 1,000$ & $26(61.9)$ \\
$\geq 500$ and $<1,000$ & $1(2.4)$ \\
$\geq 300$ and 500 & \\
Consultation & $25(59.5)$ \\
Regular conference & $5(11.9)$ \\
Meeting if necessary & $12(28.6)$ \\
Interdepartmental referral & \\
Robotic surgery & $27(64.3)$ \\
Available & $15(35.7)$ \\
Not used & \\
RT technique & $11(26.2)$ \\
Static IMRT & $10(23.8)$ \\
VMAT & \\
Tomotherapy & \\
$\geq 2$ techniques & \\
Concurrent cisplatin schedule & \\
Weekly & \\
Tri-weekly & \\
\hline$R T$ & \\
&
\end{tabular}

$\mathrm{RT}$, radiotherapy; IMRT, intensity-modulated radiotherapy; VMAT, volumetric-modulated arc therapy.

tonsil SCC with right II-III cervical LN metastasis is shown in Fig. 2A. All respondents indicated that they included the ipsilateral tonsil and involved cervical LN chain in the irradiated field, and 11 (26.2\%) also included the contralateral tonsil. The lower cervical LN chain was also included in the RT field by all respondents, and 29 (69.0\%) said that the contralateral cervical LN chain was also included.

The radiation treatment dose varied between respondents (Fig. 2B). Most prescribed $70 \mathrm{~Gy}$ and $60 \mathrm{~Gy}$ to the primary lesion and entire tonsillar fossa, respectively. In addition, 60 Gy for high-risk cervical LN chains and 50-54 Gy for low-risk chains were prescribed. Eight respondents prescribed tonsil and involved neck irradiation with $\leq 54 \mathrm{~Gy}$, which is lower than the conventional dose to high-risk areas (Fig. 2C, 2D). Cisplatin was administered as a concurrent chemotherapeutic agent and prescribed on a weekly schedule by 32 or triweekly by 10 respondents.

\section{Adjuvant RT in pT2N1M0 disease}

The extent and dose of adjuvant RT are summarized in Fig. 3A and $3 \mathrm{~B}$ in an intermediate-risk case of stage $\mathrm{pT} 2 \mathrm{~N} 1 \mathrm{M} 0$ disease with two cervical LN metastases (3-6 cm at their largest) after wide excision and neck dissection. There were no other adverse factors such as lymphovascular or perineural invasion, extranodal extension, or positive resection margins. Forty respondents (95.3\%) stated they included the tonsillar fossa, and seven (16.7\%) stated that they included the contralateral fossa in the RT field. The low-risk cervical LN chain was irradiated in 39 respondents (92.9\%) on the ipsilateral side and 19 respondents (45.2\%) on the contralateral side.

In the questionnaire regarding RT dose, the most frequently prescribed dose to the primary site and high-risk cervical LN chain was $60 \mathrm{~Gy}$; further, the dose to the low-risk cervical LN chain was diversely distributed, and the most frequently prescribed dose was 50-54 Gy (Fig. 3B). For postoperative RT, there were respondents who prescribed doses $\leq 50$ Gy with reference to ongoing clinical trials, ECOG-3311 (NCT01898494) and PATHOS (NCT02215265), associated with the de-escalation of the RT dose $[17,18]$. The de-escalated sites included the primary postoperative bed for four respondents, tonsillar fossa for eight respondents, and high-risk cervical LN chain for nine respondents. Fig. 3C-3E shows the distribution of the prescribed dose to the primary postoperative bed, tonsillar fossa, and ipsilateral involved neck chain, and de-escalation in each subsite.

\section{Definitive CCRT in ycCR after IC (initial stage T2N3M0)} The RT field and dose distribution prescribed by the respondents for definitive CCRT in ycCR in intermediate-risk cases are outlined in Fig. 4A and 4B. After IC, RT was delivered to the primary site and involved neck in all respondents, although complete remission was achieved in the follow-up imaging (Fig. 4A). The contralateral LNs tended to be included in the irradiation field with little de-escalation. The most commonly prescribed dose was 70 Gy for the primary lesion site before IC and 60 Gy for the tonsillar fossa and involved neck chain (Fig. 4B). Eight and seven respondents prescribed $\leq 54$ Gy in high-risk sites in the tonsillar fossa and in the involved neck chain, respectively (Fig. 4C, 4D). The criterion of 54 Gy was set as the dose attempted by E1308 [19], a clinical study on the de-escalation of RT dose in definitive CCRT in ycCR.

\section{Survey on considerations for de-escalation}

Forty-two radiation oncologists were asked whether de-escalation was performed with respect to the field or dose during RT in patients with HPV(+) OPC. As a result, 27 respondents (64.3\%) did not currently do so, and 15 (35.7\%) did or were considering de-escalation. 


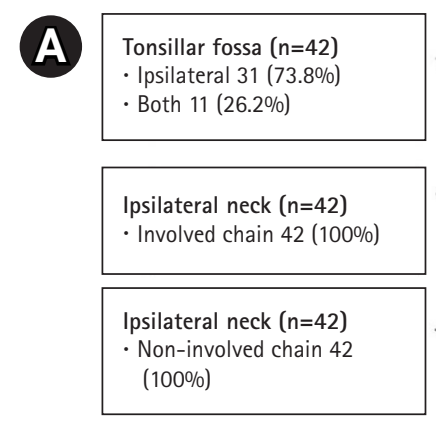

B

C

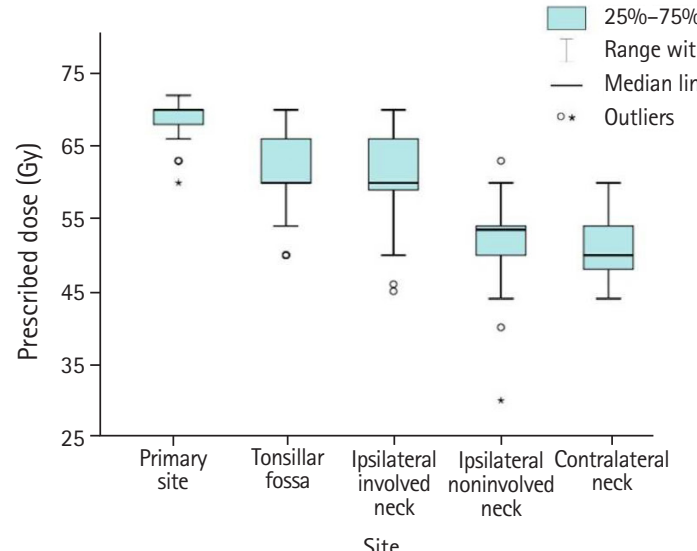

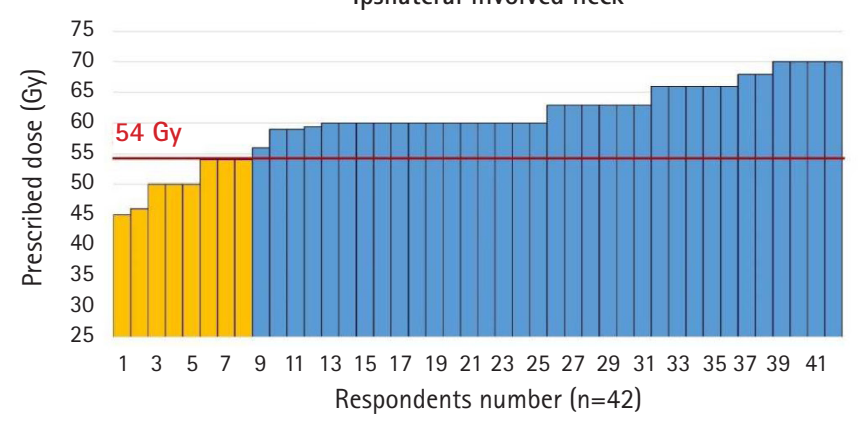

(D)

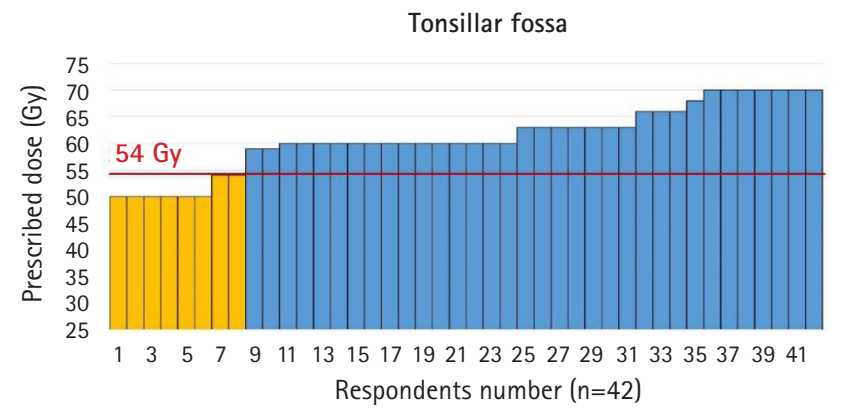

Fig. 2. Distribution of RT field (A) and prescribed doses according to the irradiated subsites shown in boxplot (B) and bar-graphs (C, D) for definitive CCRT in T2N1M0 disease. Yellow-colored bar in (C, D) represents lower doses $\leq 54 \mathrm{~Gy}$, which is lower than the conventional dose to high-risk areas. RT, radiotherapy; CCRT, concurrent chemoradiotherapy. 


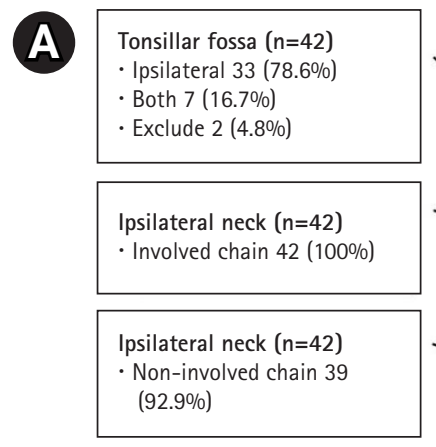

B

(C)

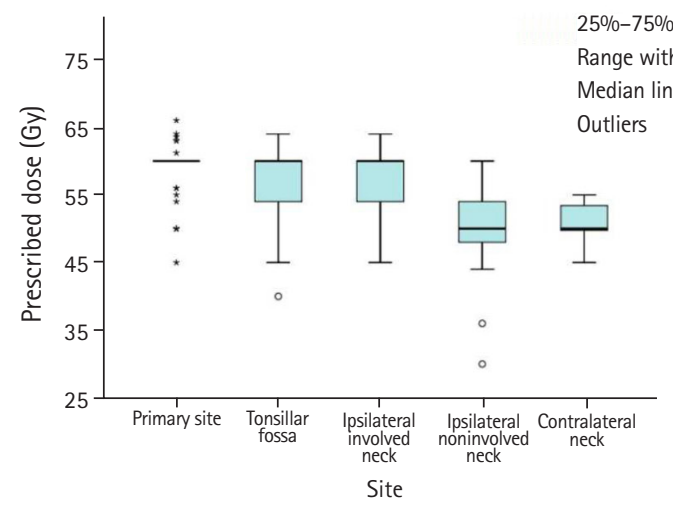

Contralateral neck $(n=42)$

- Include 19 (45.2\%)

- Exclude 23 (54.8\%)

\section{(D)}
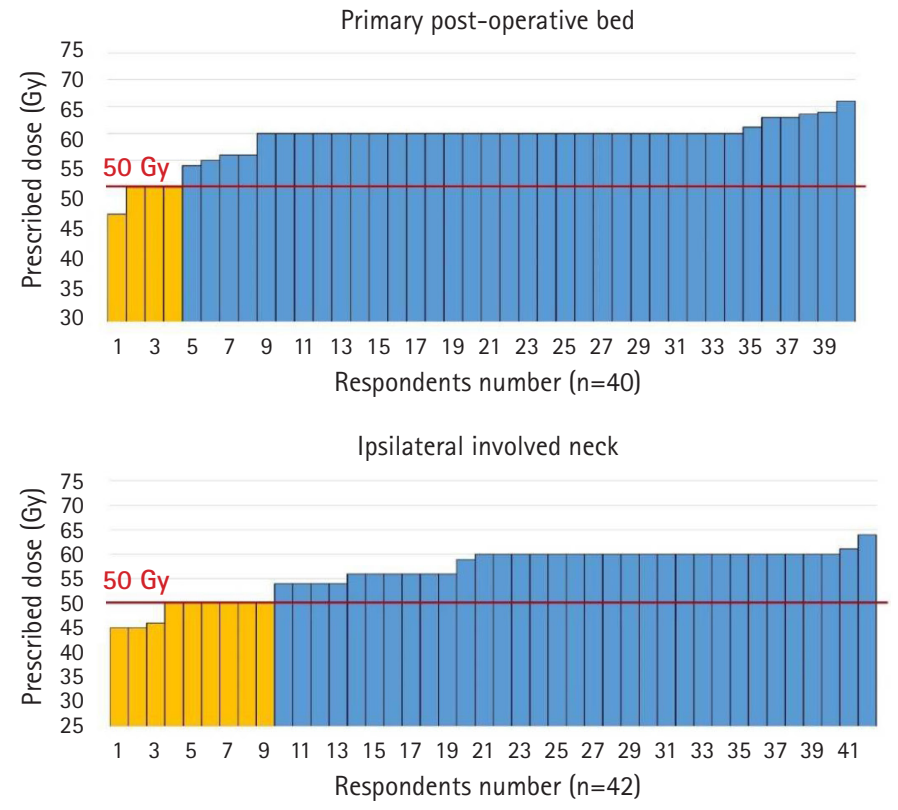

E

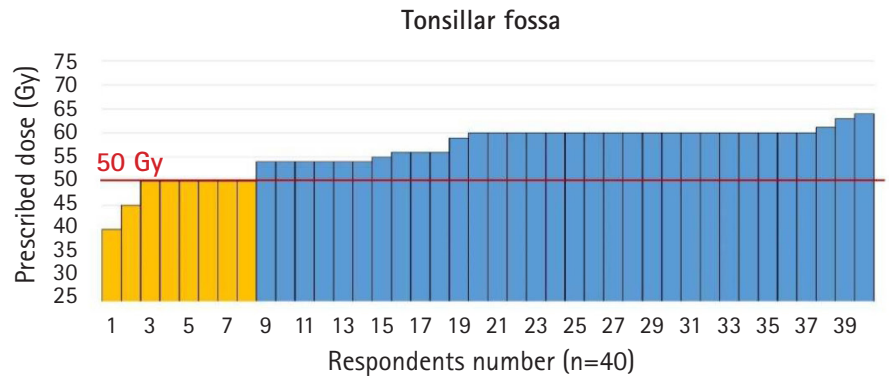

Fig. 3. Distribution of RT field (A) and prescribed doses according to the irradiated subsites shown in boxplot (B) and bar-graphs (C-E) in adjuvant RT for T2N1M0 disease. Yellow-colored bar in (C-E) represents lower doses $\leq 50$ Gy with reference to ongoing clinical trials, ECOG-3311 (NCT01898494) and PATHOS (NCT02215265), associated with the de-escalation of the RT dose. (C, E) Two respondents answered they omitted primary postoperative bed and tonsillar fossa in radiation field. RT, radiotherapy. 

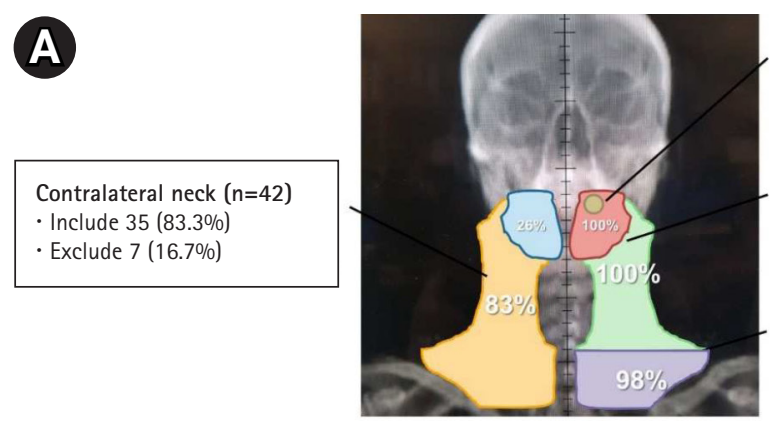

Tonsillar fossa $(n=42)$

- Ipsilateral $31(73.8 \%)$

- Both 11 (26.2\%)

Ipsilateral neck $(\mathrm{n}=42)$

- Involved chain 42 (100\%)

Ipsilateral neck $(n=42)$

- Non-involved chain 41

$(97.6 \%)$

B

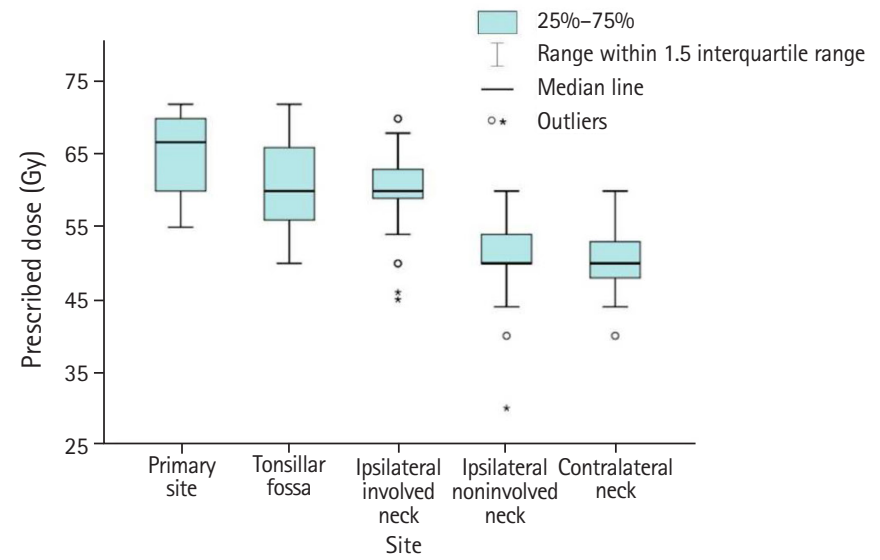

(C)

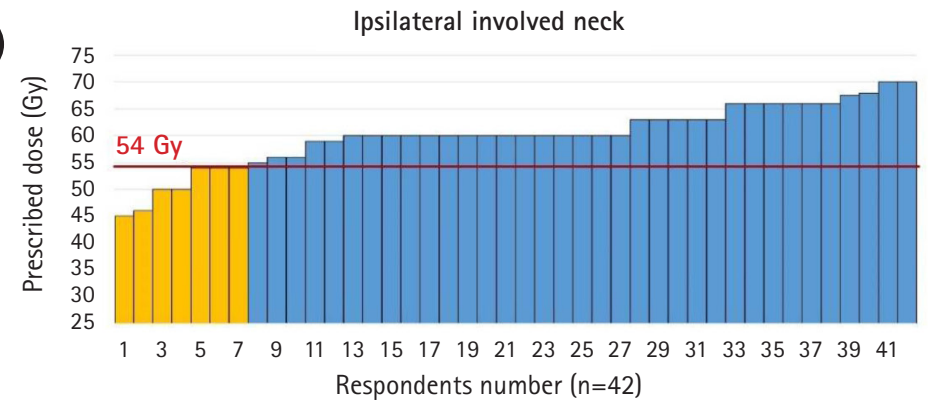

D

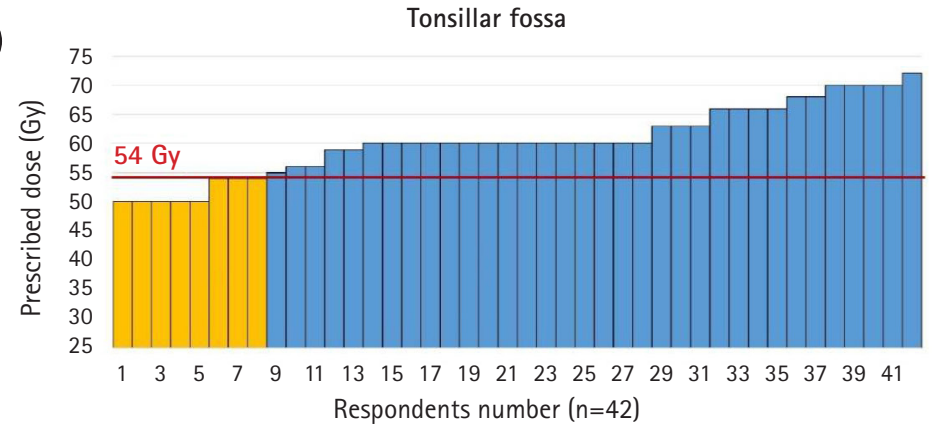

Fig. 4. Distribution of RT field (A) and prescribed doses according to the irradiated subsites shown in boxplot (B) and bar-graphs (C, D) in definitive CCRT in ycCR for initial stage T2N3M0 disease. Yellow-colored bar in (C, D) represents lower doses $\leq 54$ Gy. The criterion of $54 \mathrm{~Gy}$ was set as the dose attempted by E1308, a clinical study on the de-escalation of RT dose in definitive CCRT in ycCR. RT, radiotherapy; CCRT, concurrent chemoradiotherapy; ycCR, clinically complete remission after neoadjuvant treatment. 


\section{Discussion and Conclusion}

The incidence of $\mathrm{HPV}(+)$ OPC has rapidly increased in recent years $[20,21]$. Several investigators recognized and actually reported that more favorable locoregional control and OS outcomes were achievable among patients with $\mathrm{HPV}(+)$ OPC treated with contemporary standard treatment approaches than among patients with HPV(-) OPC $[9,21]$. This has led to controversy among many head and neck oncologists as to whether the intensity of the traditional standard treatment is optimal for $\mathrm{HPV}(+)$ patients, which has introduced the concept of "treatment de-escalation." Several attempts have been made to de-escalate treatment for HPV(+) OPC to preserve normal functional status and to maintain quality of life without compromising clinical outcomes [21].

The 8th edition of the American Joint Committee on Cancer staging system for $\mathrm{HPV}(+)$ OPC has designated lower stages than the same stage for HPV(-) OPC, unlike previous editions. These changes were due to the perception that the prognosis of patients with HPV(+) OPC was more favorable, although it appears to have had little effect on changing the treatment modality and/or its intensity. The treatment guideline by the National Comprehensive Cancer Network, however, still recommends high-dose CCRT for HPV(+) OPC patients with locoregionally advanced disease extent [22].

This questionnaire study showed interesting results regarding changes in RT protocols for HPV(+) OPC. Various studies have attempted to reduce the RT dose depending on the response, to omit chemotherapy or to replace it with immunotherapy or targeted therapy, and to sequentially administer chemoradiation instead of concurrently $[23,24]$. However, a treatment policy for RT in $\mathrm{HPV}(+)$ OPC has not yet been established. The most recently published European guidelines recommend the same treatment as that for HPV(-) OPC [25].

This study aimed to investigate current opinions regarding RT in a definitive and adjuvant setting and to assess the practical considerations of de-escalation in $\mathrm{HPV}(+)$ OPC. This is because many head and neck oncologists were asked questions regarding whether the intensity of the traditional standard treatment had been either optimal or stronger than necessary for patients with $\mathrm{HPV}(+) \mathrm{OPC}$. This questionnaire will help develop the framework of a consensus for suitable radiotherapy. It is important to examine current clinical practice before the results of ongoing prospective trials provide clear evidence.

We demonstrated reported and ongoing trials associated with the radiation field and dose in four paradigms: (1) de-escalation of chemotherapy in definitive CCRT, (2) de-escalation of the RT field and dose in definitive CCRT, (3) de-escalation of the RT field and dose in adjuvant RT after transoral surgery (TORS), and (4) omission or de-escalation of RT in complete remission after IC for locally advanced stage.

In the current questionnaire regarding definitive CCRT, approximately 20\% of respondents prescribed radiation dose reduction in the primary tonsillar fossa and high-risk cervical LN chains in $\mathrm{HPV}(+)$ OPC; however, there was no tendency to reduce the radiation field. De-escalation in the treatment of gross tumors would be risk for tumor control and survival. For this reason, there have been few studies on de-escalation of the radiation field and dose in a definitive setting. Instead of RT, there have been completed and ongoing trials on de-escalation of chemotherapy in definitive CCRT according to risk stratification. These studies can be divided into two research designs: those that do not use chemotherapy and treat with radiotherapy alone, and those that replace chemotherapeutic agents with immunotherapy. In a phase II randomized clinical study (NRG-HNOO2) of the omission of chemotherapy, it was reported that the 2-year progression-free survival (PFS) rate was $87.6 \%(p=0.2284)$ in the radiotherapy alone group, which did not meet the 2-year PFS acceptability criteria [26]. In a phase III study of immunotherapy instead of chemotherapeutic drugs in definitive CCRT (RTOG-1016, De-ESCALaTE), cetuximab as immunotherapy led to inferior OS and PFS compared with those with cisplatin $[23,24]$. The TROG 12.01 (NCT01855451) phase III randomized trial has been conducted using a similar study design to the above mentioned RTOG-1016 and De-ESCALaTE studies, and the results will be released soon.

Rather than omitting chemotherapy or replacing it with immunotherapy, there are ongoing phase II trials to reduce the radiation dose from 70 Gy to 60 Gy according to risk stratification in CCRT (NCT03077243) or reduction of the irradiated volume of the elective nodal chain (EVADER, NCT03822897). More studies are needed to determine suitable changes in the treatment for definitive CCRT. For de-escalation of chemotherapy in definitive CCRT, analysis was previously performed in a pattern of care study conducted by $\mathrm{KSH}$ NO [16], and there were no responders who substitute chemoagents, and there were some responders who omitted chemotherapy in early cancer.

De-escalation of adjuvant treatment after surgery is being discussed more frequently, and many studies are being conducted to evaluate the association between survival and de-escalation. In the AVOID phase II study [27], RT was performed only on the neck, excluding the primary lesion, in patients with no pathological adverse factors after TORS and neck dissection, and the results showed that the 2-year local control rate was $98.3 \%$ and the PFS rate was 96.2\%. During the median follow-up period of 2.4 years, none of the patients required a feeding tube associated with RT. A similar phase II study (NCT03729518) is in progress where RT avoiding the 
primary site was provided after TORS primary site resection and ipsilateral neck dissection for locally advanced HPV(+) OPC.

There are clinical studies of radiation dose reduction for adjuvant treatment after surgery in which results have been reported or ongoing. In MC1273 phase II study [28], radiation doses of $30 \mathrm{~Gy}$ and 36 Gy were prescribed according to risk stratification, and survival analysis after adjuvant CCRT was performed. The 2-year OS and PFS were $98.7 \%$ and $91.1 \%$, respectively, and a low risk of toxicity was reported. In an ongoing phase III clinical study (DART-HPV, NCT02908477), RT (30 Gy) with docetaxel and radiotherapy alone (60 Gy) were randomly assigned to patients with intermediate risk according to risk stratification.

Although relatively good local control rates were reported in the AVOID phase II study [27], there were no respondents who performed radiotherapy alone in the neck except at the primary site in the current survey. In this survey, two respondents excluded the primary site from the radiation field. Most respondents did not prescribe a very low dose of 30-36 Gy, as in the MC1273 study [28]. However, attempts to slightly reduce the dose from $60 \mathrm{~Gy}$ to $50 \mathrm{~Gy}$ in patients at intermediate risk according to risk stratification, such as in the ongoing ECOG-3311 and PATHOS trials, were observed in approximately $20 \%$ of respondents [18].

The role of IC in locally advanced HNC is not recommended, and previous studies have reported relatively low evidence of improved survival [29-33]. However, the benefit of IC has been expected to reduce distant metastasis and organ preservation in real-world practice. There have been several studies on the de-escalation of RT after IC, and PFS and toxicity were evaluated for 2-years after lowering the radiation dose according to the degree of response or risk stratification. In the phase II single-arm E1308 trial [19], IC (cisplatin, paclitaxel, and cetuximab) followed by RT (54 Gy) with cetuximab in ycCR was performed, and a 2-year PFS of $80 \%$ and OS of $94 \%$ were reported. In patients treated with reduced doses, it was found that swallowing and feeding-related toxicity were relatively low. In the Quarterback phase II study [34], CCRT was performed by reducing the dose of 56 Gy by $2: 1$ randomization after IC with docetaxel, cisplatin, and 5-fluorouracil, and the 3-year PFS and OS results were similar to those of the standard dose group. Currently, the Quarterback 2b study, a study in which CCRT was performed with RT at a dose of 56 Gy versus a standard dose according to the response to $\mathrm{IC}$, is ongoing.

In the OPTIMA phase II study [35], CCRT of 45, 50, or 75 Gy of radiation with paclitaxel, 5-fluorouracil, and hydroxyurea was provided according to risk stratification after nab-paclitaxel and carboplatin chemotherapy; the 2-year PFS rates were 95\% and 94\% in low-risk and high-risk patients, respectively. The incidence of mucositis and need for a gastrostomy tube were significantly lower in the low-dose group than in the high-dose group. The OPTIMA-II phase II study is now in progress, adding nivolumab as an induction chemotherapeutic agent and administering cisplatin as the CCRT regimen. In the current study, 18\% of respondents prescribed a radiation dose of $\leq 54 \mathrm{~Gy}$ to the high-risk neck chain. Although a large-volume phase III study remains necessary, the reduction of radiation dose to 54-56 Gy has shown reduced toxicity with no reduction in 2-year PFS in phase II studies.

In conclusion, in a survey of radiation oncologists for head and neck tumors in Korea, most of $\mathrm{HPV}(+)$ OPC treatments were performed according to the guidelines for HPV(-) OPC treatments with respect to the radiation field and dose. However, the tendency to de-escalate treatment was found in approximately 18\%-20\% of the respondents. It is necessary to establish a consensus on the radiation field and dose through multidisciplinary conferences. When de-escalation is attempted in specific patients, clinical trial enrollment is actively recommended before the evidence-based results of the abovementioned ongoing clinical studies become available.

\section{Conflict of Interest}

No potential conflict of interest relevant to this article was reported.

\section{Acknowledgments}

We thank the respondents from the following 42 institutions for participating in this survey: Asan Medical Center, Yonsei University Severance Hospital, Samsung Medical Center, Seoul National University Hospital, Gachon University Gil Medical Center, The Catholic University of Korea Seoul St. Mary's Hospital, Chungnam National University Hospital, Bundang Seoul National University Hospital, Pusan National University Hospital, Ajou University Hospital, Chonbuk National University Hospital, Korea University Anam Hospital, Dong-A University Hospital, Soonchunhyang University Bucheon Hospital, Keimyung University Dongsan Medical Center, Inha University Hospital, Soonchunhyang University Cheonan Hospital, Gyeongsang National University Hospital, Daegu Catholic University Medical Center, Ewha Womans University Medical Center, Hanyang University Seoul Hospital, Wonju Severance Christian Hospital, Chosun University Hospital, Kyung Hee University Hospital, The Catholic University of Korea Incheon St. Mary's Hospital, Yonsei University Gangnam Severance Hospital, Inje University Busan Paik Hospital, Chungbuk National University Hospital, The Catholic University of Korea St. Vincent's Hospital, Wonkwang University Hospital, The Catholic University of Korea Uijeongbu St. Mary's Hospital, Chonnam National University Hwasun Hospital, Kangbuk Sam- 
sung Hospital, The Catholic University of Korea Daejeon St. Mary's Hospital, Kyung Hee University Hospital at Gangdong, The Catholic University of Korea Bucheon St. Mary's Hospital, Presbyterian Medical Center, National Cancer Center, Inje University Ilsan Paik Hospital, Catholic Kwandong University International St. Mary's Hospital, The Catholic University of Korea Eunpyeong St. Mary's Hospital, and Korea Cancer Center Hospital.

\section{References}

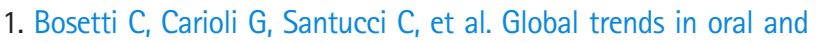
pharyngeal cancer incidence and mortality. Int J Cancer 2020; 147:1040-9.

2. Schmidt S, Linge A, Zwanenburg A, et al. Development and validation of a gene signature for patients with head and neck carcinomas treated by postoperative radio(chemo)therapy. Clin Cancer Res 2018;24:1364-74.

3. Leemans CR, Braakhuis BJ, Brakenhoff RH. The molecular biology of head and neck cancer. Nat Rev Cancer 2011;11:9-22.

4. Pytynia KB, Dahlstrom KR, Sturgis EM. Epidemiology of HPV-associated oropharyngeal cancer. Oral Oncol 2014;50:380-6.

5. Nair D, Mair M, Singh A, D'Cruz A. Prevalence and impact of human papillomavirus on head and neck cancers: review of indian studies. Indian J Surg Oncol 2018;9:568-75.

6. Vokes EE, Agrawal N, Seiwert TY. HPV-associated head and neck cancer. J Natl Cancer Inst 2015;107:djv344.

7. Fakhry C, Westra WH, Li S, et al. Improved survival of patients with human papillomavirus-positive head and neck squamous cell carcinoma in a prospective clinical trial. J Natl Cancer Inst 2008;100:261-9.

8. Rischin D, Young RJ, Fisher R, et al. Prognostic significance of p16INK4A and human papillomavirus in patients with oropharyngeal cancer treated on TROG 02.02 phase III trial. J Clin Oncol 2010;28:4142-8.

9. Ang KK, Harris J, Wheeler R, et al. Human papillomavirus and survival of patients with oropharyngeal cancer. N Engl J Med 2010;363:24-35.

10. Settle K, Posner MR, Schumaker LM, et al. Racial survival disparity in head and neck cancer results from low prevalence of human papillomavirus infection in black oropharyngeal cancer patients. Cancer Prev Res (Phila) 2009;2:776-81.

11. Lassen P, Eriksen JG, Hamilton-Dutoit S, Tramm T, Alsner J, Overgaard J. Effect of HPV-associated p16INK4A expression on response to radiotherapy and survival in squamous cell carcinoma of the head and neck. J Clin Oncol 2009;27:1992-8.

12. O'Rorke MA, Ellison MV, Murray $\sqcup$, Moran M, James J, Anderson LA. Human papillomavirus related head and neck cancer survival: a systematic review and meta-analysis. Oral Oncol 2012;48: 1191-201.

13. Mirghani $H_{1}$ Amen $F_{1}$ Tao $Y$, Deutsch $E_{1}$ Levy A. Increased radiosensitivity of HPV-positive head and neck cancers: molecular basis and therapeutic perspectives. Cancer Treat Rev 2015;41:844-52.

14. Ziemann F, Arenz A, Preising S, et al. Increased sensitivity of HPV-positive head and neck cancer cell lines to $x$-irradiation + /cisplatin due to decreased expression of E6 and E7 oncoproteins and enhanced apoptosis. Am J Cancer Res 2015;5:1017-31.

15. Kim SI, Kang JW, Noh JK, et al. Gene signature for prediction of radiosensitivity in human papillomavirus-negative head and neck squamous cell carcinoma. Radiat Oncol J 2020;38:99-108.

16. Choi KH, Song JH, Kim YS, et al. Recent treatment patterns of oropharyngeal cancer in Korea based on the expert questionnaire survey of the Korean Society for Head and Neck Oncology (KSHNO). Cancer Res Treat 2021;Jan 29 [Epub]. http://dx.doi.org/10. 4143/crt.2020.973.

17. Bigelow EO, Seiwert TY, Fakhry C. Deintensification of treatment for human papillomavirus-related oropharyngeal cancer: Current state and future directions. Oral Oncol 2020;105:104652.

18. Hargreaves S, Beasley M, Hurt C, Jones TM, Evans M. Deintensification of adjuvant treatment after transoral surgery in patients with human papillomavirus-positive oropharyngeal cancer: the conception of the PATHOS study and its development. Front Oncol 2019;9:936.

19. Marur S, Li S, Cmelak AJ, et al. E1308: Phase II trial of induction chemotherapy followed by reduced-dose radiation and weekly cetuximab in patients with HPV-associated resectable squamous cell carcinoma of the oropharynx: ECOG-ACRIN Cancer Research Group. J Clin Oncol 2017;35:490-7.

20. Kelly JR, Husain ZA, Burtness B. Treatment de-intensification strategies for head and neck cancer. Eur J Cancer 2016;68:12533.

21. Chera BS, Amdur RJ. Current status and future directions of treatment deintensification in human papilloma virus-associated oropharyngeal squamous cell carcinoma. Semin Radiat Oncol 2018;28:27-34.

22. National Comprehensive Cancer Network. NCCN Guidelines for head and neck cancer [Internet]. Plymouth Meeting, PA: National Comprehensive Cancer Network; 2021 [cited 2021 May 14]. Available from: https://www.nccn.org/professionals/physician_ gls/pdf/head-and-neck.pdf.

23. Mehanna $H_{1}$ Robinson $M$, Hartley $A$, et al. Radiotherapy plus cisplatin or cetuximab in low-risk human papillomavirus-positive oropharyngeal cancer (De-ESCALaTE HPV): an open-label randomised controlled phase 3 trial. Lancet 2019;393:51-60.

24. Gillison ML, Trotti AM, Harris J, et al. Radiotherapy plus cetux- 
imab or cisplatin in human papillomavirus-positive oropharyngeal cancer (NRG Oncology RTOG 1016): a randomised, multicentre, non-inferiority trial. Lancet 2019;393:40-50.

25. Machiels JP, Rene Leemans C, Golusinski W, et al. Squamous cell carcinoma of the oral cavity, larynx, oropharynx and hypopharynx: EHNS-ESMO-ESTRO Clinical Practice Guidelines for diagnosis, treatment and follow-up. Ann Oncol 2020;31:1462-75.

26. Yom SS, Torres-Saavedra P, Caudell JJ, et al. NRG-HN002: A randomized phase II trial for patients with p16-positive, non-smoking-associated, locoregionally advanced oropharyngeal cancer. Int J Radiat Oncol Biol Phys 2019;105:684-5.

27. Swisher-McClure S, Lukens JN, Aggarwal C, et al. A phase 2 trial of alternative volumes of oropharyngeal irradiation for de-intensification (AVOID): omission of the resected primary tumor bed after transoral robotic surgery for human papilloma virus-related squamous cell carcinoma of the oropharynx. Int J Radiat Oncol Biol Phys 2020;106:725-32.

28. Ma DJ, Price KA, Moore EJ, et al. Phase II evaluation of aggressive dose de-escalation for adjuvant chemoradiotherapy in human papillomavirus-associated oropharynx squamous cell carcinoma. J Clin Oncol 2019;37:1909-18.

29. Haddad R, O'Neill A, Rabinowits G, et al. Induction chemotherapy followed by concurrent chemoradiotherapy (sequential chemoradiotherapy) versus concurrent chemoradiotherapy alone in locally advanced head and neck cancer (PARADIGM): a randomised phase 3 trial. Lancet Oncol 2013;14:257-64.

30. Hitt R, Grau JJ, Lopez-Pousa A, et al. A randomized phase III trial comparing induction chemotherapy followed by chemoradiotherapy versus chemoradiotherapy alone as treatment of unresectable head and neck cancer. Ann Oncol 2014;25:216-25.

31. Cohen EE, Karrison TG, Kocherginsky M, et al. Phase III randomized trial of induction chemotherapy in patients with N2 or N3 locally advanced head and neck cancer. J Clin Oncol 2014;32: 2735-43.

32. Ghi MG, Paccagnella A, Ferrari $D$, et al. Induction TPF followed by concomitant treatment versus concomitant treatment alone in locally advanced head and neck cancer: a phase II-III trial. Ann Oncol 2017;28:2206-12.

33. Geoffrois L, Martin L, De Raucourt D, et al. Induction chemotherapy followed by cetuximab radiotherapy is not superior to concurrent chemoradiotherapy for head and neck carcinomas: results of the GORTEC 2007-02 phase III randomized trial. J Clin Oncol 2018;36:3077-83.

34. Misiukiewicz K, Gupta V, Miles BA, et al. Standard of care vs reduced-dose chemoradiation after induction chemotherapy in HPV+ oropharyngeal carcinoma patients: The Quarterback trial. Oral Oncol 2019;95:170-7.

35. Seiwert TY, Foster CC, Blair EA, et al. OPTIMA: a phase II dose and volume de-escalation trial for human papillomavirus-positive oropharyngeal cancer. Ann Oncol 2019;30:297-302. 